\title{
The unfolding public health crisis of transitional homelessness during the COVID-19 pandemic in India: A psychodermatologic perspective
}

\author{
Bishurul Hafi ${ }^{1}$, NA Uvais ${ }^{2}$, Muhammed Razmi T', TP Afra ${ }^{1}, K^{1}$ Silah ${ }^{3}$
}

${ }^{1}$ Consultant Dermatologist, Department of Psychodermatology, IQRAA International Hospital and Research Center, Kozhikkode, Kerala, India, ${ }^{2}$ Consultant Psychiatrist, Department of Psychodermatology, IQRAA International Hospital and Research Center, Kozhikkode, Kerala, India, ${ }^{3}$ House Surgeon, Kozhikkode Medical College, Kozhikkode, Kerala, India

Corresponding author: Dr. Bishurul Hafi, E-mail: bishuru@gmail.com

Sir,

Homelessness is a mounting worldwide public health concern. According to the 2011 census, 449,761 families in India live in open spaces, such as on pavements, under flyovers and staircases, in places of worship, and on railway platforms, with a significant proportion of them living in urban areas [1]. Migration from rural to urban areas in search of employment is considered an important reason for the significantly higher growth of the homeless population in Indian cities when compared to the rural areas [2]. Research indicates that a significant proportion of the homeless population, including women and people with multiple disabilities, engage in some kind of work for survival in the cities they live in, thereby contributing to the national economy [2].

To prevent the spread of the severe acute respiratory syndrome coronavirus 2 (SARS-CoV-2) pandemic, the Indian government enforced complete lockdown at the national level on March 24, 2020, for 21 days. Although the lockdown achieved its desired effect of flattening the epidemic curve for a short period, it precipitated a considerable social and public health crisis, mainly transitional homelessness among the migrant population. In spite of the disadvantageous financial conditions of the migrant population due to the absence of paid work, several landlords were reported to have forced them to pay the rent, ultimately leaving them with no other choice but to vacate their place of residence. Homelessness, coupled with hunger, forces them to walk for days for hundreds of kilometers together with their families, including children, back to their places of origin. Multiple images of ulcers and fissures on bare feet have surfaced in media. Many of the transitional homeless migrants were stopped at districts and state borders and quarantined in overcrowded government shelters, where social distancing is nearly impossible.

Many of these shelters also house the chronic homeless population along with migrant laborers, which increases the health risk further. Research data indicates that a significant proportion of chronic homeless people in India are suffering from some form of psychiatric illness and intellectual disability [3]. It is reasonable to speculate that, due to their psychiatric morbidities, many of them may not properly understand the magnitude of the current crisis and may not practice disinfection and social distancing even in shelters with a smaller number of occupants. Multiple studies have found that the prevalence of dermatological conditions is significantly higher among shelter home inmates with the most prevalent dermatoses infectious in nature, such as tinea pedis, body louse infestation, scabies, and impetigo [4,5]. There is an ongoing epidemic of treatment-resistant superficial mycoses in India and the process of migration and homelessness may amplify it [6]. This will, in all likelihood, increase the burden of already overworked dermatologists, aggravating their professional burnout [7].

All the above indicates that transitional homelessness has become a significant public health crisis in dealing with the COVID-19 pandemic in India, which proper planning, empathy, and adequate financial support

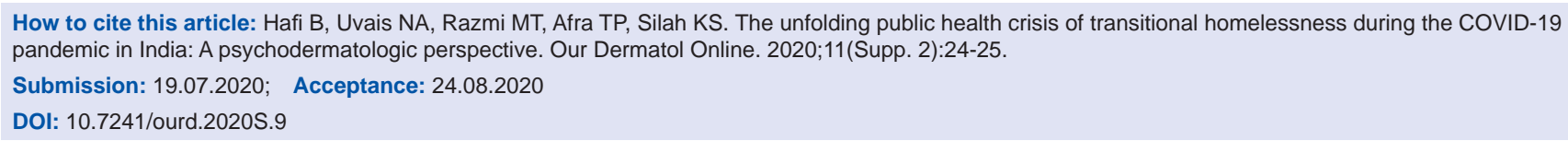


could have avoided. There are many important lessons to learn from this crisis by other developing countries fighting the COVID-19 outbreak.

\section{Consent}

The examination of the patient was conducted according to the principles of the Declaration of Helsinki.

The authors certify that they have obtained all appropriate patient consent forms, in which the patients have given consent for images and other clinical information to be included in the journal. The patients understand that their names and initials will not be published and due effort will be made to conceal their identity, but that anonymity cannot be guaranteed.

\section{REFERENCES}

1. Kaur R, Pathak RK. Homelessness and mental health in India. Lancet Psychiatry. 2016;3:500-1.
2. Singh N, Koiri P, Shukla SK. Signposting invisibles: A study of the homeless population in India. Chinese Sociological Dialogue. 2018;3:179-96.

3. Ul Hassan F, Nagavarapu LS, Prasad MK, Raj A, Sekhar K. Homelessness in mental illness: Opportunities \& prospects in the Indian context. Asian J Psychiatr. 2019;45:28-32.

4. Contag C, Lowenstein SE, Jain S, Amerson E. Survey of symptomatic dermatologic disease in homeless patients at a shelterbased clinic. Our Dermatol Online. 2017;8:133-7.

5. Badiaga S, Menard A, Dupont HT, Ravaux I, Chouquet D, Graveriau C, et al. Prevalence of skin infections in sheltered homeless. Eur J Dermatol. 2005;15:382-6.

6. Verma S, Madhu R. The great Indian epidemic of superficial dermatophytosis: An appraisal. Indian J Dermatol. 2017;62:227-36.

7. de Moll EH. Physician burnout in dermatology. Cutis. 2018;102:E24-5.

Copyright by Bishurul Hafi, et al. This is an open-access article distributed under the terms of the Creative Commons Attribution License, which permits unrestricted use, distribution, and reproduction in any medium, provided the original author and source are credited.

Source of Support: Nil, Conflict of Interest: None declared. 